\title{
International Students: A Population Facing Significant Stress
}

\author{
David Henneberry* \\ Department of Agricultural Economics, Oklahoma State University, USA \\ *Corresponding author: David Henneberry, Department of Agricultural Economics, Oklahoma State University, USA. \\ To Cite This Article: David Henneberry. International Students: A Population Facing Significant Stress. Am J Biomed Sci \& Res. 2019 - 3(2). \\ AJBSR.MS.ID.000650. DOI: 10.34297/AJBSR.2019.03.000650
}

Received: May 22, 2019 | Published: May 28, 2019

\section{Mini Review}

Stress is a condition for which both treatments, and the patient's ability to change and adapt, partially depend upon environmental factors. Some groups are particularly prone to exposure to significant stressors. The purpose of this opinion piece is to draw awareness of the significant stressors faced by a particular group of underserved individuals who bear a large burden of stressors while living almost unnoticed amongst us.

International students are prevalent on all major university campuses, medical schools, stem subject programs and graduate colleges across the United States. Institutions eagerly cash payments for tuition, lodging and academic supplies but seldom devote resources to the mental health of this group. Their level of stress is unique, because it comes from so many different areas of life, all at potentially debilitating levels [1].

Let's take a moment to reflect on the various sources of stress that this group faces.

First, and most obvious, is academic stress. International students are often in graduate programs or intensely competitive STEM fields. They come from highly competitive environments (e.g. China and India) and enter programs in the United States which then become highly competitive because of their presence.

Second, language stress is present in almost all cases. It is exhausting (both physically and mentally) to function constantly in a foreign language. Internationals in academic institutions are often affected disproportionately by language stress because the nature of their academic programs requires that they master nuances of meaning in order to excel. It is difficult to command a language at a level which allows an understanding of nuance, much less be engaged in academic competition utilizing nuance and its application in examinations and term papers [2].

Third, financial stress is a given when students are confronted with the reality of costs of education in the United States. International students do not have access to the many student loan programs which benefit students native to the United States. Nonresident tuition rates raise the cost up to $300 \%$ for international students (compared to domestic students). Furthermore, their families feel an obligation to help that is much stronger than that faced by their counterparts in the United States but are often unable to do so because of exchange rates and differences in income levels across countries.

Fourth, family stress is present in almost all families with an international student in the United States. The realities of this age group are that they have multiple life changes occurring at the same time. Families are usually a source of internal strength and support. When family members are separated by continental geography it magnifies the stress and weakens the ability of individuals to access the support functions of their nuclear and extended family.

Fifth, social stress affects international students because they are at an age where marriage and the creation of their own nuclear family unit is a priority. In the United States, dating, courtship and marriage is distinctly different than in the rest of the world. International students tend to pursue the social priorities of their home country societies - but are doing so in the highly individualistic culture of the United States. The USA has already entered the era of population decline (particularly among whites) and this social climate is tremendously different from that of most international student's home countries.

Sixth, logistic stress is a particularly difficult area for international students. How do they find housing? What are the legal implications of a lease? How do you get a driver's license? What is a "title" to an automobile? Why is insurance so expensive? How do you understand the bus schedules? These issues, which Americans almost take for granted, become large sources of stress for international students. Many arrive expecting that good public transportation will be readily available, and then discover that the car culture of the United States is so strong that in the Midwest, public transportation is almost unavailable.

Seventh, religious stress is significant for non-Christian students who are devoted to a religion which has no temples, churches or meeting halls available in their area. Many individuals 
find comfort and solace in religion. When it is suddenly unavailable, they are often unprepared to make substitute choices to fulfill the same needs. Also, many religions discourage attending a similar, but different, faith. This reduces the opportunities for coping mechanisms to develop for international students.

Eighth, dietary stress is larger than most people might imagine. When you live in a different country and many of your favorite foods are suddenly unavailable, and the composition of a meal is significantly changed, this can cause a significant stress on a human being. The timing of meals can change, the available foods change and the flavor and texture of food can change. Since many stressed out people use food as a coping mechanism, it is not uncommon to see weight loss or gain among international students. Changes in weight can be a mirror of the stress level faced by this population.

Ninth, identity stress is a reality faced by many international students. They are used to simply being treated as an individual, or a member of an extended family, in their home countries. In the United States, they may be suddenly thrust into a role of representing an entire country or race. They might be referred to as "the Mexican" or "that Asian girl". They probably never thought about themselves in that way before and bearing the responsibility of national or racial representation can be mentally exhausting, and minimizes their individual identity.

In this short opinion piece, I have identified nine areas of stress that can affect international students in the United States. Any one of these areas can create an almost debilitating level of stress. The sum of all nine areas at once can be enough to make an individual almost non-functional. Yet this group continues to function at high levels and to be academically competitive. It's hard to understand how they continue to do it. A greater awareness of their presence and the issues they face is important in order to understand the complexity of the modern society in which we live. Psychiatric services for this group are almost non-existent because of cost and insurance issues, yet they would certainly benefit from an enhanced access to mental health support services.

\section{References}

1. Sovic Sylvia (2008) “Coping with Stress: The Perspective of International Students". Art, Design and Communication in Higher Education 6(3).

2. Haber Frank, Griffiths Stephanie (2017) “5 unique mental health stressors faced by international students". European Association for International Education. 